\title{
Intermittency in Hall-magnetohydrodynamics with a strong guide field
}

P. Rodriguez Imazio, L. N. Martin, P. Dmitruk, and P. D. Mininni

Citation: Phys. Plasmas 20, 052506 (2013); doi: 10.1063/1.4807378

View online: http://dx.doi.org/10.1063/1.4807378

View Table of Contents: http://pop.aip.org/resource/1/PHPAEN/v20/i5

Published by the AIP Publishing LLC.

\section{Additional information on Phys. Plasmas}

Journal Homepage: http://pop.aip.org/

Journal Information: http://pop.aip.org/about/about_the_journal

Top downloads: http://pop.aip.org/features/most_downloaded

Information for Authors: http://pop.aip.org/authors

\section{ADVERTISEMENT}

\section{AIP Applied Physics Letters}

\section{EXPLORE WHAT'S NEW IN APL}

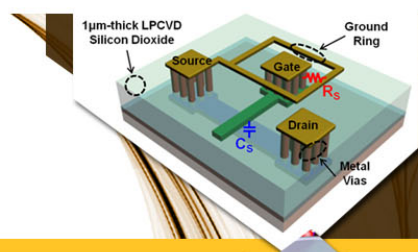

\section{SURFACES AND} INTERFACES

Focusing on physical, chemical, biological

structural, optical, magnetic and electrical

properties of surfaces and interfaces, and more.. 


\title{
Intermittency in Hall-magnetohydrodynamics with a strong guide field
}

\author{
P. Rodriguez Imazio, ${ }^{1}$ L. N. Martin, ${ }^{1}$ P. Dmitruk, ${ }^{1}$ and P. D. Mininni ${ }^{1,2}$ \\ ${ }^{1}$ Departamento de Física, Facultad de Ciencias Exactas y Naturales, Universidad de Buenos Aires and IFIBA, \\ CONICET, Buenos Aires 1428, Argentina \\ ${ }^{2}$ National Center for Atmospheric Research, P.O. Box 3000, Boulder, Colorado 80307, USA
}

(Received 24 January 2013; accepted 1 May 2013; published online 23 May 2013)

We present a detailed study of intermittency in the velocity and magnetic field fluctuations of compressible Hall-magnetohydrodynamic turbulence with an external guide field. To solve the equations numerically, a reduced model valid when a strong guide field is present is used. Different values for the ion skin depth are considered in the simulations. The resulting data are analyzed computing field increments in several directions perpendicular to the guide field, and building structure functions and probability density functions. In the magnetohydrodynamic limit, we recover the usual results with the magnetic field being more intermittent than the velocity field. In the presence of the Hall effect, field fluctuations at scales smaller than the ion skin depth show a substantial decrease in the level of intermittency, with close to monofractal scaling. (C) 2013 AIP Publishing LLC. [http://dx.doi.org/10.1063/1.4807378]

\section{INTRODUCTION}

The properties of small scales structures in magnetohydrodynamic (MHD) and Hall-MHD (HMHD) turbulence have been the subject of conflicting results and of several debates. In particular, much attention has been paid in the literature to the geometrical properties of current sheets in HMHD, as these structures are associated with magnetic flux reconnection and magnetic energy dissipation, processes of uttermost importance in astrophysics and space physics. ${ }^{1-4}$

While some numerical simulations indicate that current sheets affected by the Hall effect are wider than in MHD (see, e.g., Ref. 5), others observe thinner structures ${ }^{6}$ In all cases, the geometry of the currents sheets is changed, displaying the so-called X-type structure and reminiscent of the Sweet-Parker configuration in the MHD case, ${ }^{7}$ and changing to a double wedge shape reminiscent of the Petschek configuration when the Hall effect is relevant. ${ }^{8}$ In simulations of turbulent HMHD, it was observed that the peak of the spectrum of the current density was located at a wavenumber corresponding to the inverse of the ion skin depth. ${ }^{9-12}$ Since this peak can be associated with an average thickness of the current sheets, the effect was interpreted as a thickening of the current sheets as the Hall effect was increased. ${ }^{13}$ The result is in good agreements with experiments that indicate that the thickness of the current sheet in the presence of the Hall effect is given by the ion skin depth. ${ }^{8}$

Ref. 5 provides a possible answer to these conflicting results. In simulations of turbulent HMHD with a guide field, the authors observe that although the current sheet widens as the ion skin depth is increased, it also fragments internally into smaller filaments.

The case in which thinner structures were observed ${ }^{6}$ suggests that HMHD is more intermittent than MHD. This is also the case in some observations in the solar wind turbulence using the Cluster magnetic data. ${ }^{14,15}$ However, other observations in the solar wind of high-frequency magnetic field fluctuations from the same spacecraft indicate that while large scales are compatible with multifractal intermittent turbulence, small scales show non-Gaussian monoscaling. ${ }^{16}$

A quantification of the level of intermittency is important to understand the geometrical distribution of dissipation in a magnetofluid and a plasma, and it also can provide constraints for theories of magnetic energy dissipation and reconnection. While previous analysis of intermittency in HMHD was mostly based on the differences observed in the geometry and size of current sheets, or in the study of probability density functions (PDFs) of field increments at different scales, a precise quantification requires computation of both PDFs and of structure functions.

The study of intermittency based solely on observations of individual structures has several shortcomings. Although the formation of small scale structures can point out to an increase in the level of intermittency, there is more information that is needed to make such claim. If there are thinner structures, are these structures spatially localized? Or do they occupy more space than in the MHD case, thus, being space filling? In the former case, HMHD would be more intermittent, while in the latter case intermittency would be decreased by the Hall effect.

In this work, we present a detailed study of intermittency in the velocity and magnetic field fluctuations. Considering the solar wind as a motivation, the data for the analysis stem from numerical simulations of MHD and HMHD turbulence with a guide field. We use the reduced MHD (RMHD, Refs. 17 and 18) and reduced HMHD (RHMHD, Refs. 19 and 20) models to generate data under the approximation of a strong guide field (see also Ref. 21 for studies of kinetic plasma effects using other reduced models). Then, structure functions and PDFs of the fields are computed, for increments in the direction perpendicular to the guide field. To reduce errors, an average of the structure functions for several directions perpendicular to the guide field is computed using the $\mathrm{SO}(2)$ decomposition. ${ }^{22,23}$ Although at small scales in the solar wind several kinetic effects may play important roles, we found that a simple 
Hall magnetofluid reproduces some of the observations in Ref. 16, and that the presence of the Hall effect results in a substantial decrease in the intermittency of the velocity and magnetic fields at scales smaller than the ion skin depth. Potential limitations in the validity of HMHD from the more comprehensive framework of Vlasov-Maxwell kinetic theory can be found in Refs. 24-26. In particular, Refs. 24 and 25 show that HMHD is a valid limit of kinetic theory whenever the electron temperature is larger than the ion temperature. When this condition is not fulfilled, the dispersion relation of the waves in HMHD differs from that in kinetic theory. This can be expected since other effects become important. In spite of these limitations, the presence of the Hall effect is enough to obtain a decrease in the flow intermittency.

\section{REDUCED MHD AND HMHD MODELS}

For a compressible flow, the HMHD equations can be written (in dimensionless form) as

$$
\begin{gathered}
\frac{\partial \mathbf{u}}{\partial t}-\mathbf{u} \times \boldsymbol{\omega}=-\nabla\left(\frac{\mathbf{u}^{2}}{2}+\frac{\rho^{\gamma-1}}{M_{S}^{2}(\gamma-1)}\right)+\frac{1}{M_{A}^{2}} \frac{\mathbf{J} \times \mathbf{b}}{\rho} \\
+\nu \frac{\nabla^{2} \mathbf{u}}{\rho}+\left(\delta+\frac{1}{3} \nu\right) \frac{\nabla(\nabla \cdot \mathbf{u})}{\rho}, \\
\frac{\partial \mathbf{A}}{\partial t}-\mathbf{u} \times \mathbf{b}=-\epsilon \frac{\mathbf{J} \times \mathbf{b}}{\rho}-\nabla \phi+\eta \nabla^{2} \mathbf{A}, \\
\frac{\partial \rho}{\partial t}+\nabla \cdot(\rho \mathbf{u})=0, \\
\nabla \cdot \mathbf{A}=0 .
\end{gathered}
$$

In these equations, $\mathbf{u}$ is the velocity field, $\omega$ is the vorticity field, $\mathbf{J}$ is the current, $\mathbf{b}$ is the magnetic field, $\rho$ is the density of the plasma, and $\mathbf{A}$ and $\phi$ are, respectively, the magnetic and electric potentials. A barotropic law is assumed for the plasma, with the pressure given by $p=C \rho^{\gamma}$, where $C$ is a constant and $\gamma=5 / 3$. Equation (4) is the Coulomb gauge, which acts as a constraint that fixes the electric potential in Eq. (2). Control parameters of the system are the sonic Mach number $M_{S}$, the Alfvén Mach number $M_{A}$, the viscosities $\nu$ and $\delta$ (here we consider $\nu=\delta$ ), and the resistivity $\eta$. In our study, the most important control parameter is the Hall coefficient $\epsilon=\rho_{i i} / L$, where $\rho_{i i}$ is the ion skin depth and $L$ is the characteristic scale of turbulence. When $\epsilon=0$, the equations above result in the well known compressible MHD equations.

In the presence of a strong guide field, the equations above can be written using the reduced approximation often used in magnetohydrodynamics (see, e.g., Refs. 17 and 18). The approximation assumes that the magnetic field can be written as

$$
\mathbf{b}=B_{0} \hat{\mathbf{z}}+\mathbf{b}^{\prime}
$$

where $B_{0}$ is the intensity of the guide field, and $\mathbf{b}^{\prime}$ is such that $\left|\mathbf{b}^{\prime}\right| / B_{0} \ll 1$.

For convenience, when writing the dimensionless equations we assume, without loss of generality, that $B_{0}=1$ (in practice, the simulations have $B_{0}=8$ in dimensionless units). We then decompose the velocity and magnetic field fluctuations in terms of scalar potentials as

$$
\mathbf{u}=\nabla \times(\varphi \hat{\mathbf{z}}+f \hat{\mathbf{x}})+\nabla \psi
$$

and

$$
\mathbf{b}^{\prime}=\nabla \times(a \hat{\mathbf{z}}+g \hat{\mathbf{x}}) .
$$

Equation (7) ensures that the magnetic fields remains divergence free, while Eq. (6) gives us a compressible flow. The potentials $f$ and $g$ allow for dynamical components of the fields parallel to the guide field, and $\psi$ describes an irrotational component of the velocity field.

Then, Eqs. (1)-(4) can be written as (for the details, see, Refs. 5, 19, 20, and 27)

$$
\begin{gathered}
\frac{\partial u}{\partial t}=\frac{\partial b}{\partial z}+[\varphi, u]-[a, b]+\nu \nabla^{2} u, \\
\frac{\partial \omega}{\partial t}=\frac{\partial j}{\partial z}+[j, a]-[\omega, \varphi]+\nu \nabla^{2} \omega, \\
\frac{\partial a}{\partial t}=\frac{\partial(\varphi-\epsilon b)}{\partial z}+[\varphi, a]-\epsilon[b, a]+\eta \nabla^{2} a, \\
\frac{\partial b}{\partial t}=\beta_{p} \frac{\partial(u-\epsilon j)}{\partial z}+[\varphi, b]+\beta_{p}[u, a] \\
-\epsilon \beta_{p}[j, a]+\eta \beta_{p} \nabla^{2} b,
\end{gathered}
$$

where

$$
\begin{gathered}
u=-\partial_{y} f, \\
\omega=-\nabla_{\perp}^{2} \varphi, \\
b=-\partial_{y} g, \\
j=-\nabla_{\perp}^{2} a,
\end{gathered}
$$

and the notation $[A, B]=\partial_{x} A \partial_{y} B-\partial_{x} B \partial_{y} A$ is employed for the Poisson bracket. The potential $\psi$ was eliminated from these equations using the equation for the pressure. Finally, $\beta_{p}=\beta \gamma /(1+\beta \gamma)$ is a function of the plasma "beta." As in the previous set of equations, these equations become the compressible RMHD equations when $\epsilon=0$.

\section{NUMERICAL SIMULATIONS}

Simulations analyzed in this work are similar to those described in Ref. 5. We use a standard parallel pseudospectral code to evaluate the nonlinear terms and solve numerically the equations. ${ }^{28}$ A second-order Runge-Kutta time integration scheme is used. The magnetic field fluctuations in all simulations are less than $10 \%$ of the external magnetic field value, so we are in the range of validity of the RHMHD model.

Periodic boundary conditions are assumed in all directions of a cube of side $2 \pi L$ (where $L \sim 1$ is the initial correlation length of the fluctuations, defined as the length unit). The runs performed throughout this paper do not contain any 
magnetic or velocity external stirring terms, so the RHMHD system is let to evolve freely.

To generate the initial conditions, we excite initially Fourier modes (for both magnetic and velocity field fluctuations) in a shell in $k$-space with wavenumbers $1 \leq k \leq 2$, with the same amplitude for all modes and with random phases. Only plane-polarized fluctuations (transverse to the mean magnetic field) are excited, so the initial conditions are (low- to high-frequency) Alfvén mode fluctuations with no magnetosonic modes.

In a first set of simulations, spatial resolution is $512^{2}$ grid points in the plane perpendicular to the external magnetic field and 32 grid points in the parallel direction (this is possible because the structures that require high resolution only develop in the directions perpendicular to the field), allowing four different runs to be done with four different Hall coefficients. The kinetic and magnetic Reynolds numbers are defined, respectively, as $R=1 / \nu, R_{m}=1 / \eta$, based on unit initial r.m.s. velocity fluctuation, unit length, and dimensionless values for the viscosity and diffusivity. For all the runs, we used $R=R_{m}=1600$ (i.e., $\nu=1 / 1600, \eta$ $=1 / 1600)$. We also considered a Mach number $M_{S}=1 / 4$, and an Alfvén Mach number $M_{A}=1$.

For $\epsilon$, four values were considered, namely $\epsilon=0$ (run A, MHD), 1/32 (run B), 1/16 (run C), and 1/8 (run D). As the numerical domain used has size $2 \pi$ (see above), these values correspond, respectively, to ion skin depths with associated wavenumbers $k_{\epsilon}=\infty, 32,16$, and 8 . Data from these simulations are used for the analysis in Sec. V.

To quantify the effect of spatial resolution in the level of intermittency, runs A and D were computed also (with the same parameters) larger grids, first with spatial resolution of $768^{2}$ $\times 32$ grid points, and later with $512^{2} \times 64$ grid points. This second set of simulations (namely, runs $\mathrm{A} 2$ and $\mathrm{A} 3$, with $\epsilon=0$, and D2 and D3, with $\epsilon=1 / 8$ ) are considered in Sec. VI.

\section{MEASURES OF INTERMITTENCY}

In order to characterize velocity and magnetic field anisotropy, scaling laws, and intermittency, we present in Sec. V power spectra, structure functions, and PDFs of velocity and magnetic field increments.

The perpendicular total energy spectrum $E\left(k_{\perp}\right)$ is defined as usual, summing the power of all (velocity and magnetic) modes in Fourier space over cylindrical shells with radius $k_{\perp}$, with their axis aligned with the direction of the guide field.

To compute structure functions and PDFs, field increments must be first defined. Given the presence of the external magnetic field, it is natural to consider an axisymmetric decomposition for the increments. In general, the longitudinal increments of the velocity and magnetic fields are defined as

$$
\begin{aligned}
& \delta u(\mathbf{x}, \mathbf{l})=[\mathbf{u}(\mathbf{x}+\mathbf{l})-\mathbf{u}(\mathbf{x})] \cdot \frac{\mathbf{l}}{|\mathbf{I}|}, \\
& \delta b(\mathbf{x}, \mathbf{l})=[\mathbf{b}(\mathbf{x}+\mathbf{l})-\mathbf{b}(\mathbf{x})] \cdot \frac{\mathbf{l}}{|\mathbf{I}|},
\end{aligned}
$$

where the spatial increment $\mathbf{I}$ can point in any direction. Structure functions of order $p$ are then defined as

$$
S_{p}^{u}(\mathbf{I})=\left\langle\delta u^{p}(\mathbf{x}, \mathbf{I})\right\rangle
$$

for the velocity field, and as

$$
S_{p}^{b}(\mathbf{I})=\left\langle\delta b^{p}(\mathbf{x}, \mathbf{I})\right\rangle
$$

for the magnetic field. Here, brackets denote spacial average over all values of $\mathbf{x}$.

These structure functions depend on the direction of the increment. For isotropic and homogeneous turbulence, it is a standard practice to average over several directions, to obtain the isotropic component of the structure functions (see, e.g., Refs. 29-31). Due to the axisymmetry associated with the external magnetic field, in our case, we will be interested instead only in the increments perpendicular to $\hat{z}$. We denote increments in this direction as $\mathbf{I}_{\perp}$, and we follow the procedure explained in Refs. 22 and 23 to average over several directions of $\mathbf{I}_{\perp}$.

The method can be described as follows. Velocity and magnetic field structure functions were computed from Eqs. (18) and (19) using 24 different directions for the increments $\mathbf{l}$, generated by integer multiples of the vectors $(1,0,0),(1$, $1,0),(2,1,0),(3,1,0),(0,1,0),(-1,1,0),(1,2,0),(-2,1$, $0),(-1,2,0),(1,3,0),(-3,1,0)$, and $(-1,3,0)$ (all vectors are in units of grid points in the simulations), plus the $12 \mathrm{vec}-$ tors obtained by multiplying them by -1 . Once these structure functions were calculated, the perpendicular structure functions $S_{p}^{y}\left(l_{\perp}\right)$ and $S_{p}^{b}\left(l_{\perp}\right)$ were obtained by averaging over these 24 directions in the $x y$ plane.

For all runs, this procedure was applied to 9 snapshots of the velocity and magnetic fields, centered around the time of the peak of maximum dissipation (at $t \approx 4.5$ ), and separated by intervals $\Delta t=0.5$.

For large enough Reynolds number, the structure functions are expected to show inertial range scaling, i.e., we expect that for some range of scales $S_{p}^{u} \sim l_{\perp}^{\xi_{p}}$ and $S_{p}^{b} \sim l_{\perp}^{\zeta_{p}}$, where $\xi_{p}$ and $\zeta_{p}$ are, respectively, the scaling exponents of order $p$ of the velocity and magnetic field. As sufficient scale separation is needed to determine these exponents, in Sec. V, we show scaling exponents for runs $\mathrm{A}(\epsilon=0)$ and $\mathrm{D}$ $(\epsilon=1 / 8)$, as these runs have well defined MHD (run A) or HMHD (run D) inertial ranges. Runs B and $C$ have the ion skin depth in the middle of the inertial range, and each subrange (the MHD subrange and the HMHD subrange) is not sufficiently resolved to compute exponents.

The scaling exponents for each snapshot of the fields are obtained from the structure functions $S_{p}^{u}$ and $S_{p}^{b}$ using the least square method (extended self-similarity ${ }^{32,33^{p}}$ is not used to estimate the exponents). The values presented in Sec. V correspond to the time average over the 9 snapshots of each field. As the errors in the least square calculation are negligible when compared with the variations for each snapshot, the errors in the determination of the scaling exponents are estimated by the statistical mean square error; e.g., for the magnetic field scaling exponents the error is

$$
e_{\zeta_{p}}=\frac{1}{M} \sqrt{\sum_{i=1}^{M}\left(\zeta_{p_{i}}-\overline{\zeta_{p}}\right)^{2}},
$$


where $M=9$ is the number of snapshots of the field used in the analysis, $\zeta_{p_{i}}$ is the slope obtained from a least square fit for the $i$-th snapshot, and $\overline{\zeta_{p}}$ is the mean value averaged over all snapshots.

Finally, to complete the analysis, we consider PDFs of longitudinal increments and of derivatives of the perpendicular velocity and magnetic fields. In all cases, the PDFs are normalized by their variance, and will be shown together with a Gaussian with unit variance as a reference.

\section{RESULTS}

\section{A. Energy spectrum}

Before proceeding with the analysis of intermittency, we briefly present the total energy spectrum for all the runs with spatial resolution of $512^{2} \times 32$ grid points. This is important as determination of the inertial range based on the spectrum and on the structure functions is needed to compute the scaling exponents of the fields.

Figure 1 shows the perpendicular spectrum for the total energy (kinetic plus magnetic) in runs A, B, C, and D. In run $\mathrm{A}$, a range of wavenumbers following an approximate power law can be identified, namely from $k_{\perp} \approx 4$ to $k_{\perp} \approx 20$. This range is used to compute scaling exponents below. As a reference, we also show in Fig. 1 a Kolmogorov slope. However, it should be noted that determination of the slope of the MHD energy spectrum is beyond the interest of this
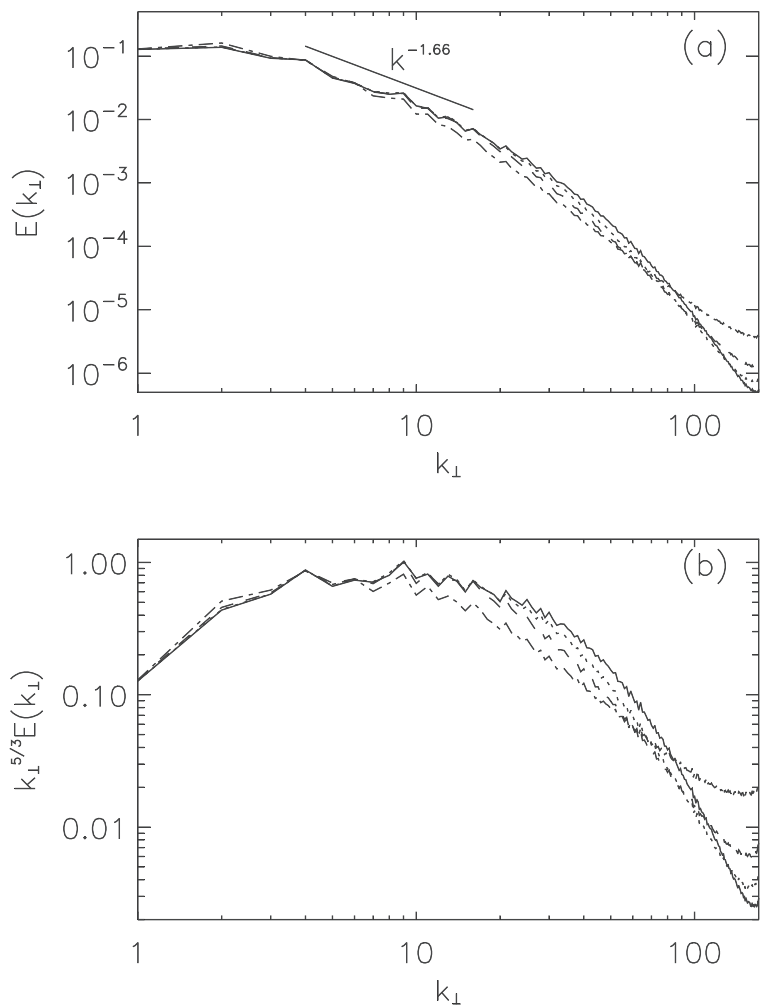

FIG. 1. (a) Perpendicular energy spectrum for runs A (solid), B (dot), C (dash), and D (dash-dot). Note how the spectrum becomes steeper in the HMHD simulations for wavenumbers larger than the inverse ion skin depth $k_{\epsilon}$ (respectively, 32, 16, and 8 for runs $\mathrm{B}, \mathrm{C}$, and D). The slope indicates Kolmogorov scaling as a reference. (b) Perpendicular energy spectrum compensated by $k^{-5 / 3}$ for the same runs. work, and readers interested in the topic are referred to detailed recent studies on the subject. ${ }^{34-36,42}$

It is also interesting to note that the energy spectrum in Fig. 1 is shallower than that expected for weak turbulence, ${ }^{37-40}$ even though the reduced equations are written for $\left|\mathbf{b}^{\prime}\right| \ll B_{0}$. Indeed, we verified that most of the energy in the simulations is in non-propagating modes (i.e., modes with $k_{\|}=0$, see Ref. 41). We also verified explicitly that the condition $\left|\mathbf{b}^{\prime}\right| / B_{0} \ll k_{\|} / k_{\perp}$ is violated, where $k_{\|}$and $k_{\perp}$ are characteristic parallel and perpendicular wavenumbers (obtained from the flow parallel and perpendicular integral scales). This condition is required in RMHD for weak turbulence to develop, ${ }^{39}$ and as a result we will interpret the following results in the framework of strong turbulence.

As the value of $\epsilon$ in the simulations is increased (see runs B, C, and D in Fig. 1), the spectrum becomes steeper at wavenumbers larger than $k_{\epsilon}$. This trend has been observed before in simulations, ${ }^{13,43,47}$ and it has been argued that it can result in an inertial range in the HMHD subrange of the form $E(k)$ $\sim k^{-7 / 3}$ (Refs. 15, 44, and 48) (such a spectrum has also been observed in the solar wind, Refs. 16, 45, and 46). Run D has a HMHD subrange wide enough to compute structure functions and scaling exponents (from $k_{\perp}=1 / \epsilon=8$ to $k_{\perp} \approx 20$ ), while runs $\mathrm{B}$ and $\mathrm{C}$ are intermediate between runs $\mathrm{A}$ and $\mathrm{D}$ and have two barely resolved subranges. However, these two intermediate runs will be useful to study trends in the behavior of the PDFs and of the structure functions as $\epsilon$ is increased.

\section{B. Structure functions and scaling exponents}

We present here the results for the computation of the axisymmetric structure functions for the longitudinal component of the velocity and magnetic field for runs A, B, C, and D.

Figure 2 shows the structure functions for the magnetic field fluctuations up to sixth order for runs A and D. The structure functions show a range of scales with approximately power law scaling at intermediate scales, and at the smallest scales approach, the $\sim l^{p}$ scaling expected for a smooth field in the dissipative range. The velocity field structure functions (not shown) display a similar behavior, at the same range of scales. The inertial range identified in the energy spectrum $E\left(k_{\perp}\right)$ is consistent with the range of scales where $S_{p}^{u}$ and $S_{p}^{b}$ show an approximate power law behavior.

From the structure functions, the scaling exponents can be computed. Exponents for the velocity and the magnetic field up to sixth order in runs A and D are shown in Fig. 3. The range of scales used for the calculation corresponds to the subranges indicated before, with $l_{\perp}=2 \pi / k_{\perp}$. For $\epsilon=0$ (run A), the deviation of the exponents $\xi_{p}$ and $\zeta_{p}$ from a straight line is an indication of intermittency and of multifractality. In the HMHD case $(\epsilon=1 / 8$, run $\mathrm{D})$, the exponents are closer to a straight line, indicating less intermittency. In fact, within error bars and up to $p=3$, the data are consistent with $\xi_{p}=\xi_{2} p / 2$ and $\zeta_{p}=\zeta_{2} p / 2$, and therefore with monoscaling as also observed for high-frequency magnetic fluctuations in the solar wind. ${ }^{16}$

Run D (with $\epsilon=1 / 8$ ) has second scaling exponents $\xi_{2}$ and $\zeta_{2}$ which are larger than $2 / 3$ (the value expected for 

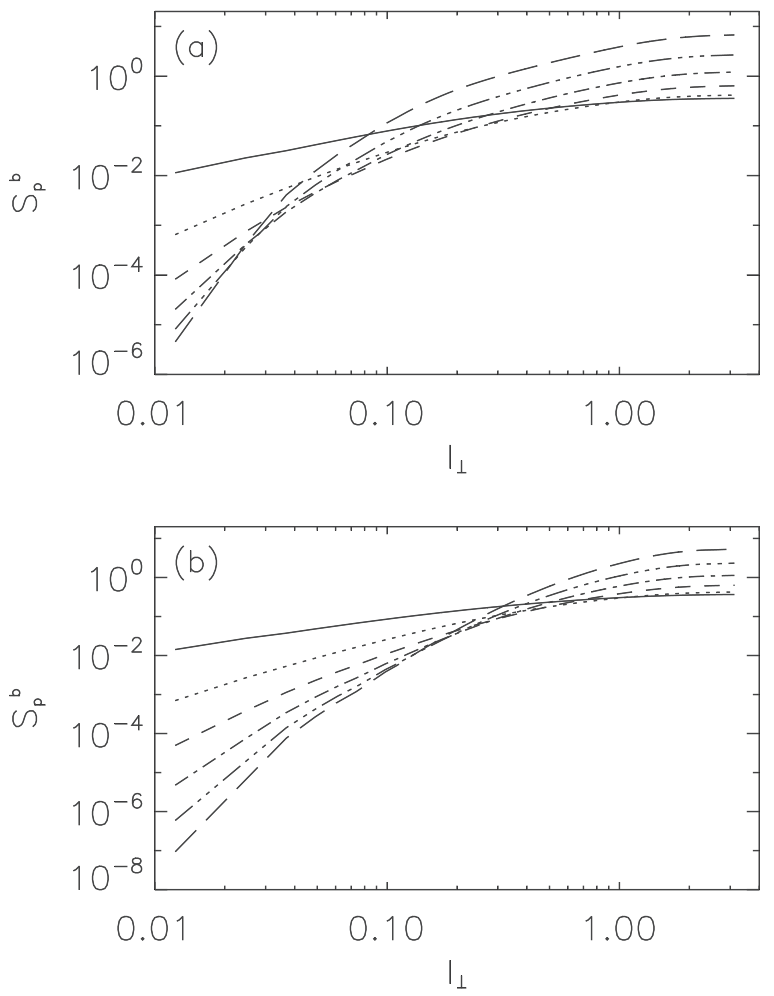

FIG. 2. Axisymmetric structure functions for the longitudinal magnetic field up to six order for (a) run A $(\epsilon=0)$, and (b) run $\mathrm{D}(\epsilon=1 / 8)$. The order of the structure function is indicated as follows: $p=1$ (solid), 2 (dot), 3 (dash), 4 (dash-dot), 5 (dash-triple-dot), and 6 (long dash).
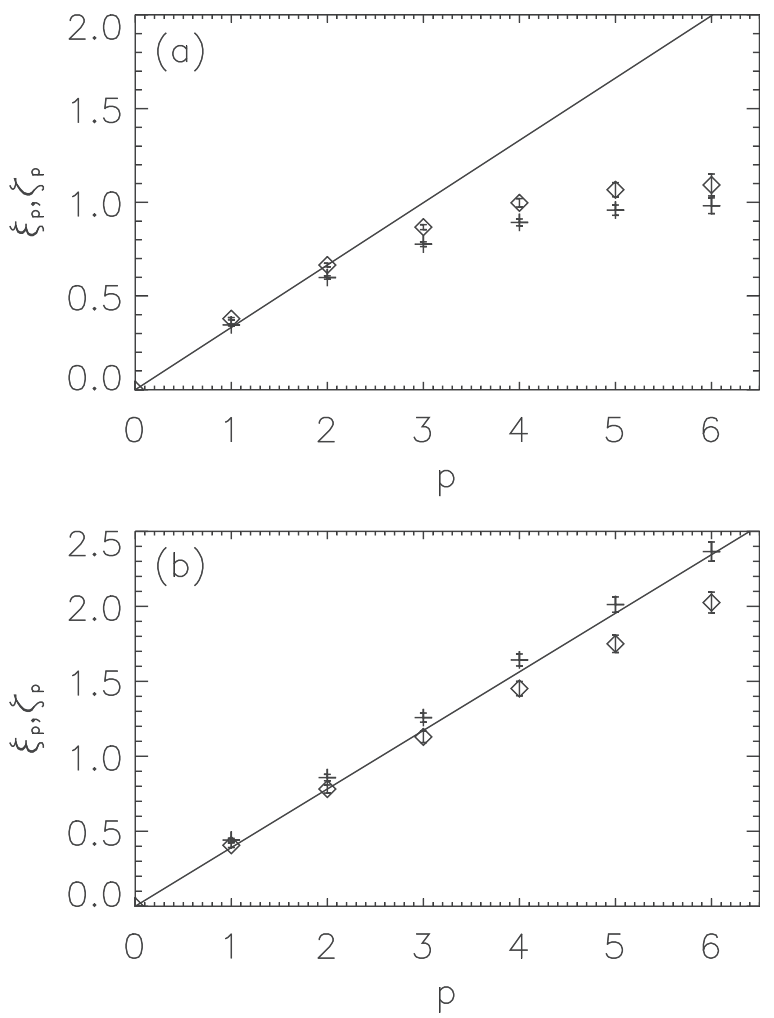

FIG. 3. Scaling exponents (with error bars) as a function of the order $p$ up to sixth order, for the velocity (crosses), and for the magnetic field (diamonds), (a) for run $\mathrm{A}(\epsilon=0)$, and (b) for run $\mathrm{D}(\epsilon=1 / 8)$. Linear scaling of the exponents with $\zeta_{2} p / 2$ (corresponding to non-intermittent scaling with the second order exponent consistent with the scaling of the energy spectrum in Fig. 1) is indicated in both cases by the straight line.
Kolmogorov scaling), but which are smaller than $4 / 3$ (the value expected if the spectrum is $\sim k^{-7 / 3}$, Refs. 49-51). This can be related to the fact that the total energy spectrum in Fig. 1 becomes steeper as the amplitude of the Hall effect is increased, but is still slightly shallower than $-7 / 3$ for the run with $\epsilon=1 / 8$. Also, note that the scaling of the velocity field and of the magnetic field can differ from that of the total energy, as is known to be the case in MHD. ${ }^{35,52}$

The deviation from strict scale invariance (linear scaling) in Fig. 3 can be quantified in terms of the intermittency exponents $\mu^{u}=2 \xi_{3}-\xi_{6}$ and $\mu^{b}=2 \zeta_{3}-\zeta_{6}$. The larger these exponent, the more intermittent the fields. For run A, these exponents are $\mu^{u}=0.57 \pm 0.07$ for the velocity field, and $\mu^{b}=0.64 \pm 0.08$ for the magnetic field. It is interesting to point out that these values that indicate that the magnetic field is more intermittent than the velocity field, are consistent with observations of large-scale fluctuations in the solar wind (see, e.g., Ref. 53), and with numerical simulations of MHD turbulence at higher spatial resolution. ${ }^{52}$

The intermittency exponents are substantially reduced for run $\mathrm{D}$, with $\mu^{u}=0.15 \pm 0.12$ for the velocity field and $\mu^{b}=0.23 \pm 0.14$ for the magnetic field. This confirms that intermittency is substantially decreased in the presence of the Hall effect.

At the spatial resolution used in these runs, the lack of sufficient scale separation in the MHD and HMHD subranges for intermediate values of $\epsilon$ does not allow the calculation of scaling exponents for runs B and C. However, the structure functions for these runs show a behavior intermediate between runs $\mathrm{A}$ and $\mathrm{D}$, and consistent with the behavior of the spectrum in Fig. 1. In other words, as the Hall coefficient $\epsilon$ is increased, the structure functions steepen at scales smaller than the ion-skin depth. As an example of this behavior, Fig. 4 shows the fourth order structure function for the magnetic field for runs A, B, C, and D. Note that runs B and $\mathrm{C}$ show a behavior consistent with the behavior of run $\mathrm{A}$ at large scales (scales larger than the ion-skin depth), and display a steeper slope (compatible with that found for run D) at smaller scales.

The results confirm that the presence of the Hall term steepens the scaling of the energy spectrum (and consistently, of the structure functions), and also show that the Hall effect reduces intermittency in the velocity and magnetic

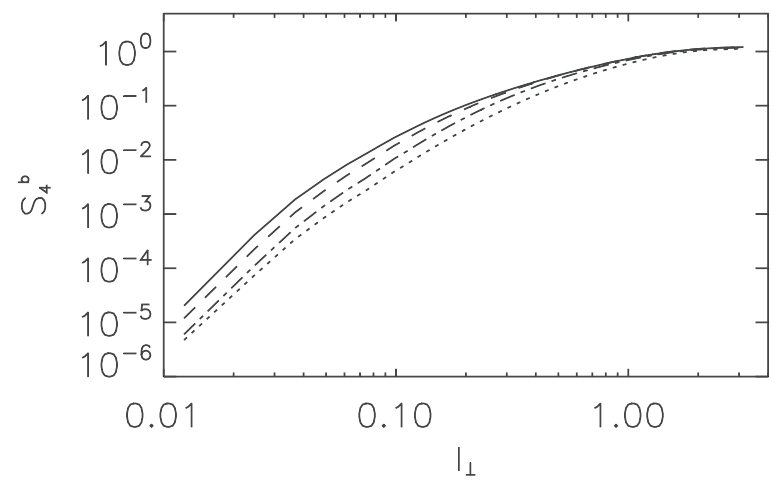

FIG. 4. Fourth order structure function of longitudinal magnetic field increments for runs A ( $\epsilon=0$, solid line), B ( $\epsilon=1 / 32$, dashed line), $\mathrm{C}(\epsilon=1 / 16$, dashed-dotted line), and $\mathrm{D}(\epsilon=1 / 8$, dotted line). 
fields. The velocity and magnetic field scaling exponents approach a linear behavior characteristic of a self-similar (non-intermittent) flows. In Sec. V C, this result is confirmed by an analysis of PDFs of velocity and magnetic field increments and spatial derivatives.

\section{Probability density functions}

We now consider PDFs for longitudinal increments of the $x$-component of the velocity and magnetic fields. As already mentioned, the PDFs will be presented normalized by their variance, and together with a Gaussian distribution with unit variance as a reference. Deviations from Gaussianity, or increase of the deviations from Gaussianity as smaller increments are considered, are a signature of intermittency.

Figure 5 shows the PDFs of the magnetic field increments for four different spatial increments, namely $l=1.6$, $0.8,0.4,0.2$, and 0.1 , for runs $\mathrm{A}, \mathrm{B}, \mathrm{C}$, and D. For all runs, the PDFs of magnetic field increments are close to Gaussian for $l=1.6$, while for smaller spatial increments nonGaussian tails and asymmetry develop. This is a common feature for many turbulent flows, with large scales close to Gaussian statistics and smaller scales developing deviations from Gaussianity with strong tails (i.e., with extreme gradients more probable than what can be expected from a normal distribution). As a reference, the integral scale in all runs (the scale with the energy containing eddies) is close to the size of the domain, $L \approx 2 \pi$, while the dissipative scale is $L_{\eta} \approx 0.05$. Increments with $l=1.6$ are close to the flow integral scale, increments with $l=0.8$ or 0.4 are in the inertial range, while $l=0.1$ is close to the dissipation length scale.

Although all runs develop non-Gaussian tails, when comparing the PDFs of the four runs with different values of $\epsilon$, it is clear that the amplitude of these tails is drastically reduced as the value of $\epsilon$ is increased. Moreover, for the largest value of $\epsilon$ considered, we cannot identify a clear increase in the amplitude of the tails as we look at smaller increments. This tendency (which is monotonic with increasing $\epsilon$ ) of the PDFs of different spatial increments to collapse into a single curve, with weaker tails than in the MHD case, is an indication of reduced intermittency and expected for scaleinvariant flows.

Figure 6 shows the same PDFs for increments of the velocity field. Again, the PDFs are close to Gaussian for the largest increment in the four runs, and non-Gaussian tails develop with increasing amplitude for smaller increments. In this case, for $\epsilon=1 / 8$ all the PDFs seem to collapse into the Gaussian, and the tails are weaker than for the magnetic field. This is consistent with the previous observation, using the intermittency coefficients $\mu^{u}$ and $\mu^{b}$, that the magnetic field is more intermittent than the velocity field, and that both fields are less intermittent in HMHD than in MHD.

To quantify the deviations from a Gaussian distribution in each run, we calculated the skewness and the kurtosis of the $x$-derivatives of the $x$-components of the velocity and magnetic fields. Note these quantities correspond, respectively, to the third- and fourth-moments of the PDFs in Figs. 5 and 6 in the limit of vanishing spatial increment. The skewness and kurtosis of a function $f$ are defined as $S(f)$
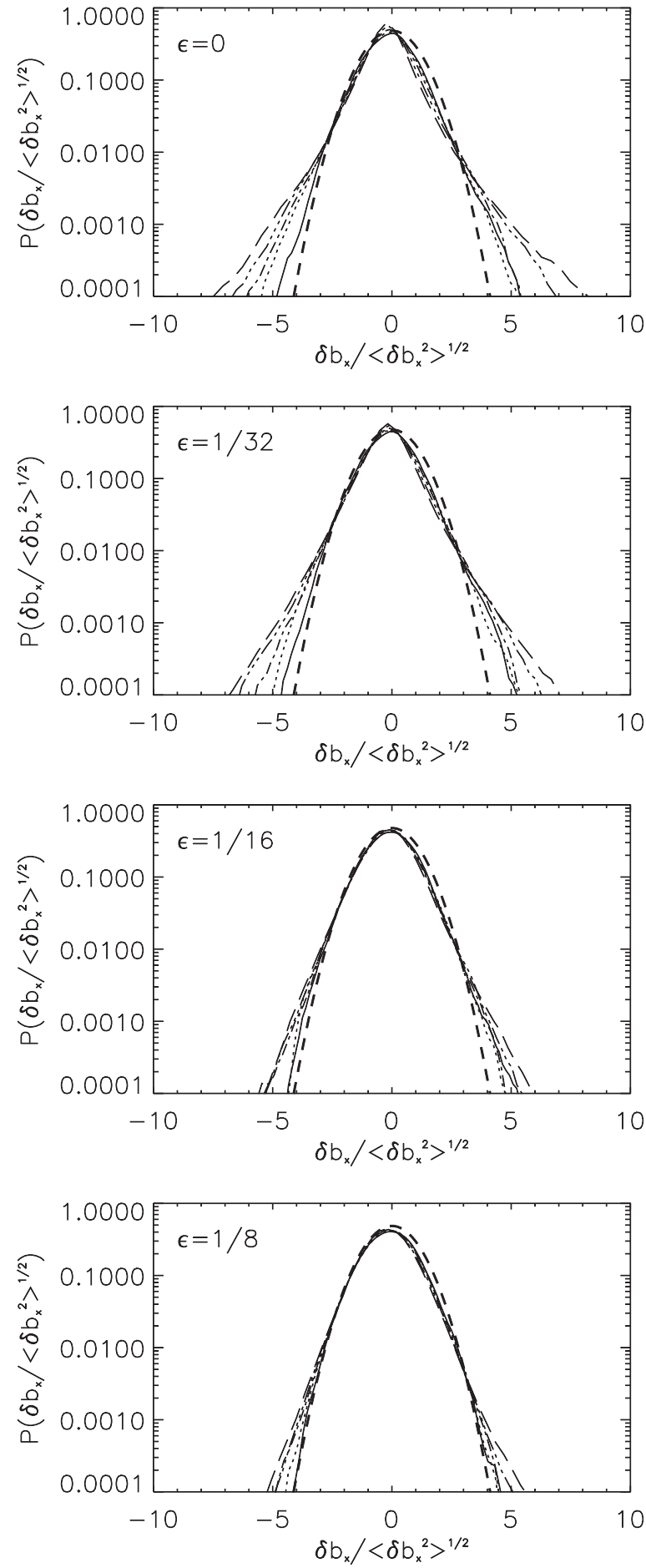

FIG. 5. PDFs for magnetic field increments, for $l=1.6$ (solid), 0.8 (dot), 0.4 (dash-dot), 0.2 (dash-triple-dot), and 0.1 (long dashes), and for runs A, B, C, and $\mathrm{D}$ from top to bottom, respectively. In all the figures, a dashed curve indicates a Gaussian PDF with unit variance.

$=\left\langle f^{3}\right\rangle /\left\langle f^{2}\right\rangle^{3 / 2}$ and $K(f)=\left\langle f^{4}\right\rangle /\left\langle f^{2}\right\rangle^{2}$, respectively, where $f$ can be, e.g., some component of the velocity (or magnetic) field gradient. The resulting values are listed in Table I. In accordance with what can be expected from a visual inspection of Figs. 5 and 6 , the skewness of $\partial_{x} u_{x}$ and $\partial_{x} b_{x}$ is reduced to almost zero for $\epsilon=1 / 8$, which indicates a substantial reduction in the asymmetry of the PDF. The kurtosis of $\partial_{x} u_{x}$ and $\partial_{x} b_{x}$ also decreases with increasing $\epsilon$, which 

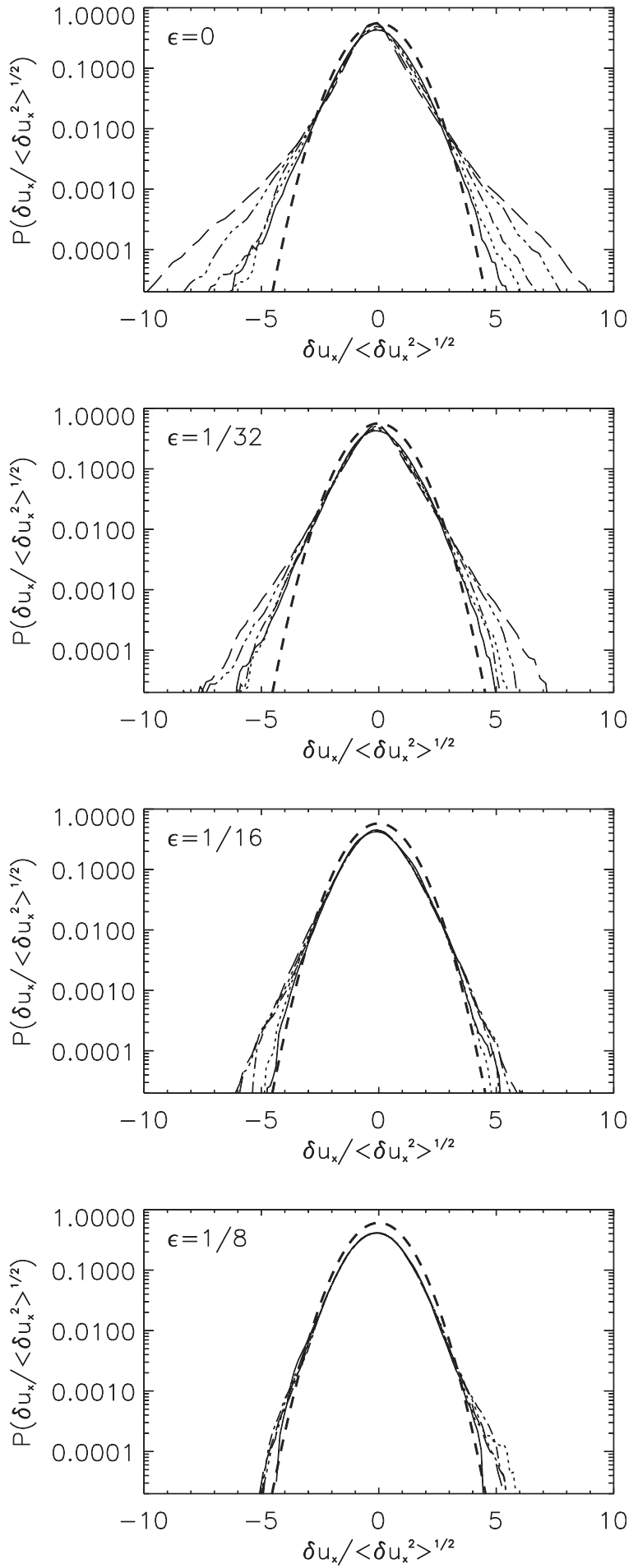

FIG. 6. PDFs for velocity field increments, for $l=1.6$ (solid), 0.8 (dot), 0.4 (dash-dot), 0.2 (dash-triple-dot), and 0.1 (long dashes), and for runs A, B, C, and $\mathrm{D}$ from top to bottom, respectively. In all the figures, a dashed curve indicates a Gaussian PDF with unit variance.

indicates a smoothing in the peakedness of the PDFs and a decrease in the intensity of the tails.

\section{EFFECT OF RESOLUTION}

Recently, it was stressed the need of using well resolved numerical simulations to quantify high order statistics and intermittency in MHD. ${ }^{54}$ In particular, it has been claimed that if the flow is not properly resolved, a partial
TABLE I. Skewness $(S)$ and kurtosis $(K)$ for the $x$-derivatives of $b_{x}$ and $u_{x}$, for all runs with spatial resolution $512^{2} \times 32$ and with different amplitudes of the Hall effect $\epsilon . S\left(\partial_{x} b_{x}\right)$ and $K\left(\partial_{x} b_{x}\right)$ are, respectively, the skewness and kurtosis of the magnetic field spatial derivatives, while $S\left(\partial_{x} u_{x}\right)$ and $K\left(\partial_{x} u_{x}\right)$ are the corresponding quantities for the velocity field derivatives.

\begin{tabular}{lcccc}
\hline \hline Quantity & $\epsilon=0$ & $\epsilon=1 / 32$ & $\epsilon=1 / 16$ & $\epsilon=1 / 8$ \\
\hline$S\left(\partial_{x} u_{x}\right)$ & -0.18 & -0.013 & -0.01 & -0.001 \\
$K\left(\partial_{x} u_{x}\right)$ & 19 & 8.1 & 5 & 4.9 \\
$S\left(\partial_{x} b_{x}\right)$ & 0.36 & 0.17 & 0.11 & 0.07 \\
$K\left(\partial_{x} b_{x}\right)$ & 26 & 15.7 & 6.6 & 5.8 \\
\hline \hline
\end{tabular}

thermalization of the small scales may result in artificial Gaussian statistics and an artificial decrease of the intermittency. Considering this, in this section, we present results for simulations with the same parameters as in runs A and D, but with larger parallel or perpendicular spatial resolution. We consider two runs with $768^{2} \times 32$ grid points, namely, runs $\mathrm{A} 2$ and $\mathrm{D} 2$, and two runs with $512^{2} \times 64$ grid points, runs $\mathrm{A} 3$ and $\mathrm{D} 3$. Runs $\mathrm{A} 2$ and $\mathrm{A} 3$ have the same parameters and initial conditions as run $\mathrm{A}$, while runs D2 and D3 are the same as run $\mathrm{D}$ except for the change in resolution.

We computed structure functions, scaling exponents, and PDFs for runs A2, D2, A3, and D3 and compared the results with those found for runs $\mathrm{A}$ and $\mathrm{D}$. In all cases, the results were consistent within error bars. As an illustration, in Fig. 7, we show the velocity field and magnetic field scaling exponents for runs $\mathrm{D}, \mathrm{D} 2$, and $\mathrm{D} 3$ (all with $\epsilon=1 / 8$, and
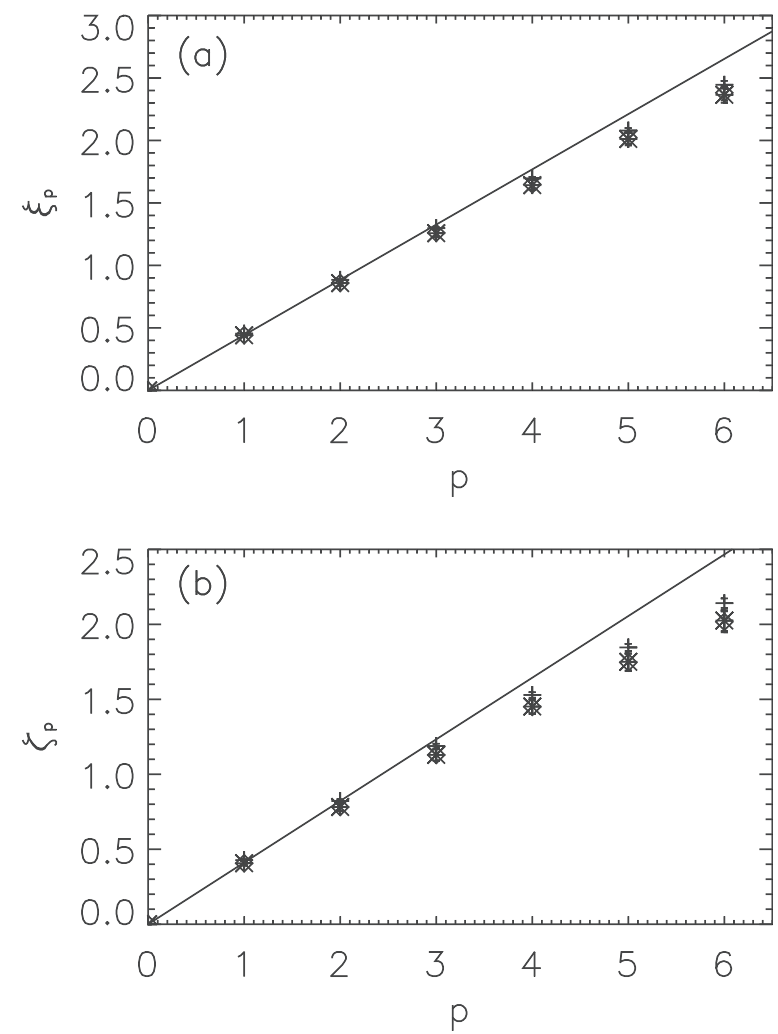

FIG. 7. (a) Velocity field scaling exponents (with error bars) as a function of the order $p$ up to sixth order, for runs D (stars), D2 (crosses), and D3 (diamonds) all with $\epsilon=1 / 8$. Linear scaling of the exponents is indicated as a reference. (b) Same for the magnetic field scaling exponents. 
respectively, with $512^{2} \times 32,768^{2} \times 32$, and $512^{2} \times 64$ grid points). Increasing the parallel or perpendicular resolution neither does change the scaling exponents nor does it change the fact that the exponents are close to the straight line and less intermittent than in the MHD case.

In run $\mathrm{A} 2$, the intermittency exponents are $\mu^{u}$ $=0.52 \pm 0.08$ and $\mu^{b}=0.70 \pm 0.07$, while in run $\mathrm{A} 3$, we obtain $\mu^{u}=0.57 \pm 0.07$ and $\mu^{b}=0.64 \pm 0.08$, consistent within error bars with the values found in run A. In run D2, the intermittency exponents are $\mu^{u}=0.15 \pm 0.04$ and $\mu^{b}=0.24 \pm 0.06$, while in run D3 the exponents are $\mu^{u}=$ $0.14 \pm 0.12$ and $\mu^{b}=0.24 \pm 0.14$, also consistent with the values obtained in run $D$.

Figure 8 shows the PDFs of velocity and magnetic field increments in runs D, D2, and D3, for a spatial increment $l=0.1$. The PDFs are almost indistinguishable. Similar results were obtained for runs $\mathrm{A}, \mathrm{A} 2$, and $\mathrm{A} 3$. When computing the PDFs of spatial derivatives of the fields, we obtained $S\left(\partial_{x} u_{x}\right)=-0.19, S\left(\partial_{x} b_{x}\right)=0.41, K\left(\partial_{x} u_{x}\right)=18$, and $K\left(\partial_{x} b_{x}\right)$ $=26$ for run $\mathrm{A} 2$, and $S\left(\partial_{x} u_{x}\right)=-0.18, S\left(\partial_{x} b_{x}\right)=0.36$, $K\left(\partial_{x} u_{x}\right)=19$, and $K\left(\partial_{x} b_{x}\right)=27$ for run A3. For the runs with Hall effect, we found $S\left(\partial_{x} u_{x}\right)=-0.001, S\left(\partial_{x} b_{x}\right)=0.08$, $K\left(\partial_{x} u_{x}\right)=8.4$, and $K\left(\partial_{x} b_{x}\right)=6.5$ for run D2, and $S\left(\partial_{x} u_{x}\right)$ $=-0.001, S\left(\partial_{x} b_{x}\right)=0.07, K\left(\partial_{x} u_{x}\right)=5.9$, and $K\left(\partial_{x} b_{x}\right)=5.8$ for run D3. These values should be compared with the values in Table I for runs A and D at lower resolution.

Wan et al. ${ }^{54}$ argue that for a MHD simulation to be well resolved, the kurtosis of the current should remains independent of the spatial resolution. In our MHD and HMHD runs that condition is fulfilled, at least up to the level of statistical fluctuations that can be expected when comparing
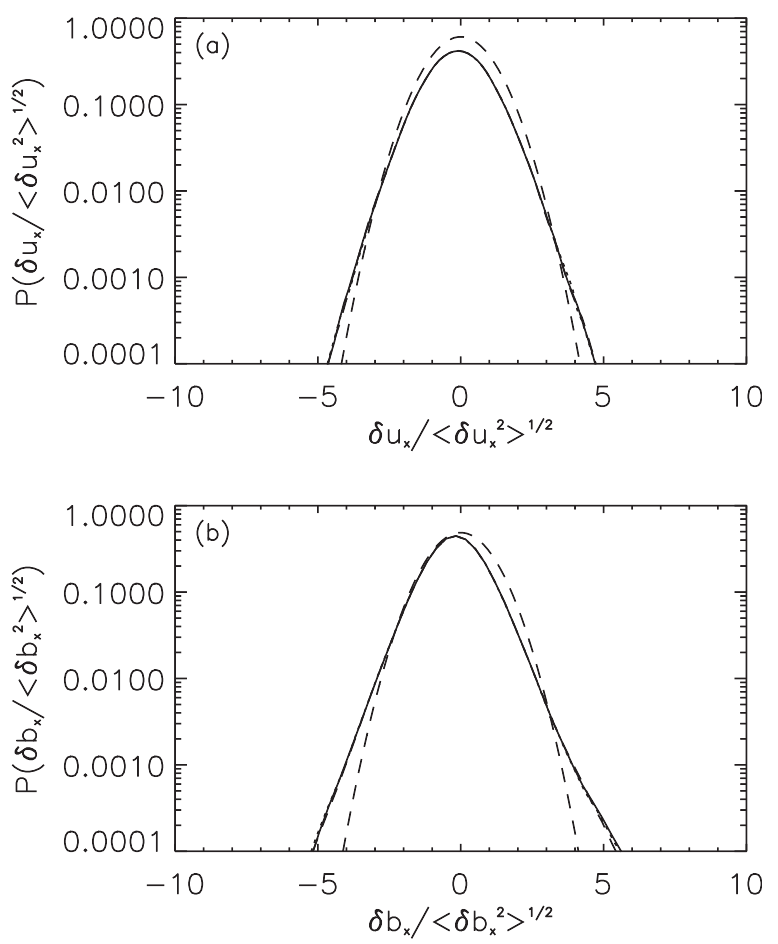

FIG. 8. (a) PDFs of velocity field increments for $l=0.1$ and $\epsilon=1 / 8$, for runs D (solid line), D2 (dotted line), and D3 (dashed-dotted line). The three PDFs are practically indistinguishable. The dashed line shows a Gaussian distribution as a reference. (b) Same for magnetic field increments. two simulations of a turbulent flow. To verify this, we computed the skewness and kurtosis of the component of the current density parallel to the external magnetic field, i.e., $S\left(j_{z}\right)$ and $K\left(j_{z}\right)$. In the MHD simulations $(\epsilon=0)$, we obtained $S\left(j_{z}\right)=0,70$ and $K\left(j_{z}\right)=21$ in the simulation with $512^{2}$ $\times 32$ grid points, $S\left(j_{z}\right)=0,71$ and $K\left(j_{z}\right)=22$ in the simulation with $768^{2} \times 32$ grid points, and $S\left(j_{z}\right)=-0,70$ and $K\left(j_{z}\right)=21$ in the simulation with $512^{2} \times 64$ grid points. In the HMHD simulations with $\epsilon=1 / 8$, we obtained $S\left(j_{z}\right)$ $=-0,02$ and $K\left(j_{z}\right)=4.5$ in the simulation with $512^{2} \times 32$ grid points, $S\left(j_{z}\right)=-0,01$ and $K\left(j_{z}\right)=4.8$ in the simulation with $768^{2} \times 32$ grid points, and $S\left(j_{z}\right)=-0,02$ and $K\left(j_{z}\right)$ $=4.5$ in the simulation with $512^{2} \times 64$ grid points.

Although there is a small increase in $S\left(j_{z}\right)$ and $K\left(j_{z}\right)$ as the perpendicular resolution is increased in both the MHD and HMHD runs, the increase is smaller than $10 \%$ in most cases. Increasing the parallel resolution seems to have no significative effect. As a result, we conclude that the simulations are well resolved even with the more stringent criteria of Wan et al ${ }^{54}$ Moreover, the reduction of the intermittency in presence of the Hall term is also confirmed by the skewness and kurtosis of the current at both spatial resolutions.

As a result, we conclude that increasing resolution has no significant effect on the results we reported in Sec. V, and that the decrease in the intermittency of the flow presented above has its source in the Hall effect and not in a numerical artifact when the flow is not properly resolved.

\section{SUMMARY AND CONCLUSIONS}

In this work, we presented a study of intermittency in the velocity and magnetic field fluctuations of compressible Hall-magnetohydrodynamic turbulence with an external guide field. Unlike previous works, we were not interested in the characterization of geometrical properties or in the size of individual structures in the flow (e.g., current sheets), but rather interested in their overall statistical properties.

The equations were solved numerically using a reduced model valid when a strong guide field is present, and both structure functions and probability density functions of field increments were computed. In the magnetohydrodynamic limit, we recovered results found in the previous studies, with the magnetic field being more intermittent than the velocity field. However, in the presence of the Hall effect, we found field fluctuations at scales smaller than the ion skin depth to be substantially less intermittent, with close to scale-invariant scaling.

As the intensity of the Hall effect was increased in the simulations (i.e., the ion skin depth was made larger in units of the box size), we found both the total energy spectrum and the structure functions to develop a steeper scaling in a wider subinertial range, for all scales smaller than the ion skin depth. The behavior of the scaling exponents for both the velocity and the magnetic field up to sixth order becomes closer to monofractal as the Hall effect is increased, and the intermittency exponent decreases accordingly.

In agreement with these results, the probability density functions of longitudinal velocity and magnetic field increments have weaker non-Gaussian tails and less asymmetry at 
scales smaller than the ion skin depth. For velocity and magnetic field gradients, the skewness and kurtosis also decrease as the Hall effect is increased.

The results were obtained for simulations with spatial resolution of $512^{2} \times 32$ grid points, and verified in simulations at larger spatial resolution, with $768^{2} \times 32$ grid points and with $512^{2} \times 64$ grid points. As a result, we can safely conclude that increasing the resolution has no effect on the results, and that the decrease in the intermittency of the flow has its source in the Hall effect.

It is interesting that although a decrease in the intermittency has been observed in high-frequency magnetic field fluctuations in the solar wind, ${ }^{15,16,55}$ the observations also show PDFs with strong non-Gaussian tails. These nonGaussian tails are absent in our simulations, and considering the limitations of the HMHD model we are led to conjecture that their origin in the solar wind lies in other kinetic effects that are not captured by our model (see, e.g., Refs. 55 and 56).

\section{ACKNOWLEDGMENTS}

P.D. and P.D.M. acknowledge support from the Carrera del Investigador Científico of CONICET. The authors acknowledge support from Grant Nos. PICT 2011-1626 and 20111529, PIP 11220090100825, and UBACYT 20020110200359.

${ }^{1}$ P. Dmitruk and W. H. Matthaeus, Phys. Plasmas 13, 042307 (2006).

${ }^{2}$ J. Birn and M. Hesse, J. Geophys. Res. 106, 3737, doi:10.1029/ 1999JA001001 (2001)

${ }^{3}$ Y. Ren, M. Yamada, S. Gerhardt, H. Ji, R. Kulsrud, and A. Kuritsyn, Phys. Rev. Lett. 95, 55003 (2005).

${ }^{4}$ M. Shay, J. F. Drake, B. N. Rogers, and R. E. Denton, J. Geophys. Res. 106, 3759, doi:10.1029/1999JA001007 (2001).

${ }^{5}$ L. N. Martin, P. Dmitruk, and D. O. Gómez, Phys. Plasmas 19, 052305 (2012).

${ }^{6}$ S. Donato, S. Servidio, P. Dmitruk, V. Carbone, M. A. Shay, and P. A. Cassak, Phys. Plasmas 19, 092307 (2012).

${ }^{7}$ S. Servidio, W. H. Matthaeus, M. A. Shay, P. A. Cassak, and P. Dmitruk, Phys. Rev. Lett. 102, 115003 (2009).

${ }^{8}$ M. Yamada, Y. Ren, H. Ji, J. Breslau, S. Gerhardt, R. Kulsrud, and A. Kuritsyn, Phys. Plasmas 13, 052119 (2006).

${ }^{9}$ P. D. Mininni, D. O. Gómez, and S. M. Mahajan, Astrophys. J. 567, L81 (2002).

${ }^{10}$ P. D. Mininni, D. O. Gómez, and S. M. Mahajan, Astrophys. J. 584, 1120 (2003).

${ }^{11}$ P. D. Mininni, D. O. Gómez, and S. M. Mahajan, Astrophys. J. 619, 1019 (2005).

${ }^{12}$ P. D. Mininni, A. Alexakis, and A. Pouquet, J. Plasma Phys. 73, 377 (2007).

${ }^{13}$ D. O. Gómez, P. D. Mininni, and P. Dmitruk, Phys. Rev. E 82, 036406 (2010).

${ }^{14}$ O. Alexandrova, V. Carbone, P. Veltri, and L. Sorriso-Valvo, Planet. Space Sci. 55, 2224 (2007).

${ }^{15}$ O. Alexandrova, V. Carbone, P. Veltri, and L. Sorriso-Valvo, Astrophys. J. 674, 1153 (2008).

${ }^{16}$ K. H. Kiyani, S. C. Chapman, Yu. V. Khotyaintsev, M. W. Dunlop, and F. Sahraoui, Phys. Rev. Lett. 103, 075006 (2009).
${ }^{17}$ D. Montgomery, Phys. Scr. T2, 83 (1982).

${ }^{18}$ H. R. Strauss, Phys. Fluids 19, 134 (1976).

${ }^{19}$ D. O. Gómez, S. M. Mahajan, and P. Dmitruk, Phys. Plasmas 15, 102303 (2008).

${ }^{20}$ L. N. Martin, P. Dmitruk, and D. O. Gomez, Phys. Plasmas 17, 112304 (2010).

${ }^{21}$ J. Cho and A. Lazarian, Astrophys. J. 701, 236 (2009).

${ }^{22}$ P. Rodriguez Imazio and P. D. Mininni, Phys. Rev. E 83, 066309 (2011).

${ }^{23}$ P. D. Mininni and A. Pouquet, Phys. Fluids 22, 035106 (2010).

${ }^{24}$ A. Ito, A. Hirose, S. M. Mahajan, and S. Ohsaki, Phys. Plasmas 11, 5643 (2004).

${ }^{25}$ G. G. Howes, Nonlinear Processes Geophys. 16, 219 (2009).

${ }^{26}$ A. A. Schekochihin, S. C. Cowley, W. Dorland, G. W. Hammett, G. G. Howes, E. Quataert, and T. Tatsuno, Astrophys. J., Suppl. 182, 310 (2009).

${ }^{27}$ N. H. Bian and D. Tsiklauri, Phys. Plasmas 16, 064503 (2009).

${ }^{28}$ S. Ghosh, M. Hossain, and W. H. Matthaeus, Comput. Phys. Commun. 74, 18 (1993).

${ }^{29}$ I. Arad, B. Dhruva, S. Kurien, V. S. L'vov, I. Procaccia, and K. R. Sreenivasan, Phys. Rev. Lett. 81, 5330 (1998).

${ }^{30}$ L. Biferale and I. Procaccia, Phys. Rep. 414, 43 (2005).

${ }^{31}$ L. N. Martin and P. D. Mininni, Phys. Rev. E 81, 016310 (2010).

${ }^{32}$ R. Benzi, S. Ciliberto, C. Baudet, G. Ruiz Chavarria, and R. Tripiccione, Europhys. Lett. 24, 275 (1993).

${ }^{33}$ R. Benzi S. Ciliberto R. Tripiccione C. Baudet, F. Massaioli, and S. Succi, Phys. Rev. E 48, R29 (1993).

${ }^{34}$ E. Lee, M. E. Brachet, A. Pouquet, P. D. Mininni, and D. Rosenberg, Phys. Rev. E 81, 016318 (2010).

${ }^{35}$ P. D. Mininni and A. Pouquet, Phys. Rev. Lett. 99, 254502 (2007).

${ }^{36}$ J. C. Perez, J. Mason, S. Boldyrev, and F. Cattaneo, Phys. Rev. X 2 041005 (2012)

${ }^{37}$ S. Galtier, S. V. Nazarenko, A. C. Newell, and A. Pouquet, J. Plasma Phys. 63, 447 (2000).

${ }^{38}$ S. Galtier and A. Bhattacharjee, Phys. Plasmas 10, 3065 (2003).

${ }^{39}$ S. Nazarenko, Wave Turbulence (Springer, Berlin, 2011).

${ }^{40}$ A. Verdini and R. Grappin, Phys. Rev. Lett. 109, 025004 (2012).

${ }^{41}$ P. Dmitruk and W. H. Matthaeus, Phys. Plasmas 16, 062304 (2009).

${ }^{42}$ P. Dmitruk, D. O. Gómez, and W. H. Matthaeus, Phys. Plasmas 10, 3584 (2003).

${ }^{43}$ S. Galtier and E. Buchlin, Astrophys. J. 656, 560 (2007).

${ }^{44}$ G. G. Howes, J. M. TenBarge, W. Dorland, E. Quataert, A. A. Schekochihin, R. Numata, and T. Tatsuno, Phys. Rev. Lett. 107, 035004 (2011).

${ }^{45}$ O. Alexandrova, J. Saur, C. Lacombe, A. Mangeney, J. Mitchell, S. J. Schwartz, and P. Robert, Phys. Rev. Lett. 103, 165003 (2009).

${ }^{46}$ F. Sahraoui, M. L. Goldstein, P. Robert, and Yu. V. Khotyaintsev, Phys. Rev. Lett. 102, 231102 (2009).

${ }^{47}$ S. Ghosh, E. Siregar, D. A. Roberts, and M. L. Goldstein, J. Geophys. Res. 101, 2493, doi:10.1029/95JA03201 (1996).

${ }^{48}$ S. Galtier, Phys. Rev. E 77, 015302 (2008).

${ }^{49}$ E. Camporeale and D. Burgess, Astrophys. J 730, 114 (2011).

${ }^{50}$ R. Meyrand and S. Galtier, Phys. Rev. Lett. 109, 194501 (2012).

${ }^{51}$ M. Wan, W. H. Matthaeus, H. Karimabadi, V. Roytershteyn, M. Shay, P. Wu, W. Daughton, B. Loring, and S. C. Chapman, Phys. Rev. Lett. 109, 195001 (2012)

${ }^{52}$ P. D. Mininni and A. Pouquet, Phys. Rev. E. 80, 025401(R) (2009).

${ }^{53}$ J. J. Podesta, D. A. Roberts, and M. L. Goldstein, Astrophys. J. 664, 543 (2007).

${ }^{54}$ M. Wan, S. Oughton, S. Servidio, and W. H. Matthaeus, Phys. Plasmas 17, 082308 (2010).

${ }^{55}$ K. H. Kiyani, S. C. Chapman, F. Sahraoui, B. Hnat, O. Fauvarque, and Yu. V. Khotyaintsev, Astrophys. J. 763, 10 (2013).

${ }^{56}$ D. Perrone, F. Valentini, S. Servidio, S. Dalena, and P. Veltri, Astrophys. J. 762, 99 (2013) 